ISSN 0975-3303

Mapana J Sci, 10, 1(2011), 52-62

https:/ / doi.org/ 10.12725/ mjs.18.5

\title{
Split and Non-Split Dominator Chromatic Numbers and Related Parameters
}

\author{
K. Kavitha, ${ }^{*}$ N.G. David ${ }^{\dagger}$ and N. Selvi ${ }^{\ddagger}$
}

\begin{abstract}
A proper graph coloring is defined as coloring the nodes of a graph with the minimum number of colors without any two adjacent nodes having the same color. Dominator coloring of $\mathrm{G}$ is a proper coloring in which every vertex of $G$ dominates every vertex of at least one color class. In this paper, new parameters, namely strong split and non-split dominator chromatic numbers and block, cycle, path non-split dominator chromatic numbers are introduced. These parameters are obtained for different classes of graphs and also interesting results are established.
\end{abstract}

Keywords: domination, dominator, split domination, non-split domination, strong split domination, block non-split domination, path non-split domination, block non-split domination, cycle nonsplit domination

\section{Preliminaries}

In our study, we consider only simple and undirected graphs. In this section, we review the notions of domination, coloring and dominator coloring $[1,2]$.

*Department of Mathematics, Madras Christian College, Chennai 600059

tDept of Mathematics, Madras Christian College; ngdmcc@gmail.com

$\ddagger$ Department of Mathematics, A.D.M. College for Women Nagapattinam, Tamil Nadu - 611 001; dr.selvinatarajan@ymail.com 
Definition 1.1 Let $G=(V, E)$ be a graph. A subset $D$ of $V$ is called a dominating set of $\mathrm{G}$ if every vertex in $\mathrm{V}-\mathrm{D}$ is adjacent to at least one vertex in $\mathrm{D}$. The domination number $\gamma(\mathrm{G})$ is the minimum cardinality of a dominating set of $\mathrm{G}$.

Definition 1.2 A proper coloring of a graph G is an assignment of colors to the vertices of $G$ in such a way that no two adjacent vertices receive the same color. The chromatic number $\chi(\mathrm{G})$, is the minimum number of colors required for a proper coloring of G. A Color class is the set of vertices, having the same color. The color class corresponding to the color $\mathrm{i}$ is denoted by $\mathrm{C}_{\mathrm{i}}$.

Definition 1.3 A dominator coloring of a graph $G$ is a proper coloring in which every vertex of $G$ dominates every vertex of at least one color class. The convention is that if $\{\mathrm{v}\}$ is a color class, then $\mathrm{v}$ dominates the color class $\{\mathrm{v}\}$. The dominator chromatic number $\chi_{\mathrm{d}}(\mathrm{G})$ is the minimum number of colors required for a dominator coloring of $\mathrm{G}$.

\section{Strong Split Dominator chromatic number}

In this section, we extend the notion of strong split domination [3], to strong split dominator chromatic number $\chi_{\text {ssd }}(G)$ and obtain $\chi_{\text {ssd }}(G)$ for the classes of graphs: trees, cycles, paths, wheels, complete graphs and bipartite graphs.

Definition 2.1 Consider a graph $\mathrm{G}$ and its dominator coloring with $\chi_{\mathrm{d}}(\mathrm{G})$ colors. The strong split dominator chromatic number of $\mathrm{G}$ is minimum number of color classes whose removal from $G$ leaves the remaining graph totally disconnected and is denoted by $\chi_{\text {ssd }}(G)$. 
Example 2.2 Let $G$ be the graph with $V=\left\{v_{1}, v_{2}, v_{3}, v_{4}, v_{5}, v_{6}\right\}$ in Figure 1.

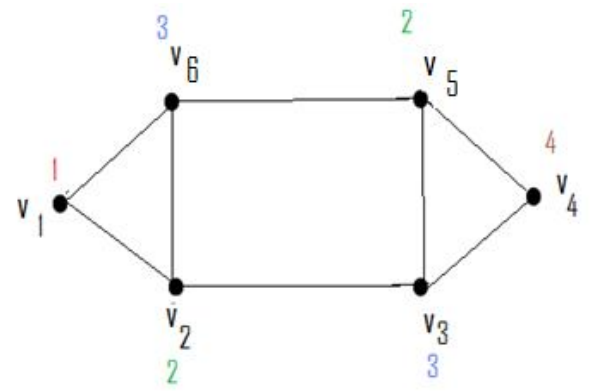

Figure 1

The color classes of $G$ are: $C_{1}=\left\{v_{1}\right\}, C_{2}=\left\{v_{2}, v_{5}\right\}, C_{3}=\left\{v_{3}, v_{6}\right\}$ and $C_{4}$ $=\left\{\mathrm{V}_{4}\right\}$.

Here, $V-\left\{C_{2}, C_{3}\right\}$ is totally disconnected. Hence $\chi_{\text {ssd }}(G)=2$.

Theorem 2.3 For cycle graphs $C_{n}$ of order $n \geq 3$,

$$
\chi_{\text {ssd }}\left(C_{n}\right)=\left\{\begin{array}{ccc}
1 & \text { when } & \mathrm{n}=4,6,8,10 \\
2 & \text { when } & \mathrm{n}=3,5,7 \\
2 & \text { when } & \mathrm{n}=3 \mathrm{k} \text { or } 3 \mathrm{k}+2, \mathrm{k} \geq 3 \\
3 & \text { when } & \mathrm{n}=3 \mathrm{k}+1, \mathrm{k} \geq 4
\end{array}\right.
$$

Proof: Let $C_{n}$ be the cycle graph of order $n \geq 3$ and let $v_{1}, v_{2}, \ldots, v_{n}$ be the label of its vertices. When $n=3,5$ or 7 , the vertices of $C_{n}$ are colored by the coloring sequence $(1,2,3),(1,2,1,3,2),(1,2,1,3,1$, $4,5)$ to obtain a dominator coloring. Hence $\chi_{\text {ssd }}\left(C_{n}\right)=2$, when $n=3$, 5 , or 7 .

Case (i) When $\mathrm{n}=4,6,8$ or 10 .

Color the odd subscripted vertices $v_{1}, v_{3}, v_{5}, \ldots$ by color 1 and the even subscripted vertices $v_{2}, v_{4}, v_{6}, \ldots$ by colors $2,3,4, \ldots$ respectively, resulting in a dominator coloring. Removal of color class 1 results in a totally disconnected graph. Hence $\chi_{\text {ssd }}\left(C_{n}\right)=1$, when $\mathrm{n}=4,6,8$ or 10 . 
Case (ii) When $\mathrm{n}=3 \mathrm{k}$, where $\mathrm{k} \geq 3$

Color the vertices $v_{1}, v_{4}, v_{7}, \ldots$ by color $1, v_{2}, v_{5}, v_{8}, \ldots$ by color 2 and $\mathrm{v}_{3}, \mathrm{v}_{6}, \mathrm{v}_{9}, \ldots$ by colors $3,4,5, \ldots$ respectively. Removal of color classes 1 and 2 results in totally disconnected graph. Hence $\chi_{\text {ssd }}\left(C_{n}\right)=2$ in this case.

Case (iii) When $\mathrm{n}=3 \mathrm{k}+1, \mathrm{k} \geq 4$

Color the vertices $\mathrm{v}_{1}, \mathrm{v}_{4}, \mathrm{v}_{7}, \ldots, \mathrm{v}_{\mathrm{n}-3}$ by color $1, \mathrm{v}_{2}, \mathrm{v}_{5}, \mathrm{v}_{8}, \ldots$, $\mathrm{v}_{\mathrm{n}-2}$ by color $2, \mathrm{v}_{3}, \mathrm{v}_{6}, \mathrm{v}_{9}, \ldots, \mathrm{v}_{\mathrm{n}-1}$ by colors $3,4,5, \ldots(\mathrm{k}+2)$ respectively and $\mathrm{v}_{\mathrm{n}}$ by color $(\mathrm{k}+3)$. Removal of color classes 1,2 and $(k+3)$ results in totally disconnected graph. Hence $\chi_{\text {ssd }}\left(C_{n}\right)=3$ in this case.

Case (iv) When $\mathrm{n}=3 \mathrm{k}+2, \mathrm{k} \geq 3$

Color the vertices $\mathrm{v}_{1}, \mathrm{v}_{4}, \mathrm{v}_{7}, \ldots, \mathrm{v}_{\mathrm{n}-1}$ by color $1, \mathrm{v}_{2}, \mathrm{v}_{5}, \mathrm{v}_{8}, \ldots$, $\mathrm{v}_{\mathrm{n}-3}$ by color $2, \mathrm{v}_{3}, \mathrm{v}_{6}, \mathrm{v}_{9}, \ldots, \mathrm{v}_{\mathrm{n}-2}$ by colors $3,4,5, \ldots(\mathrm{k}+2)$ respectively and $\mathrm{v}_{\mathrm{n}}$ by color $(\mathrm{k}+3)$. Removal of color classes 1,2 results in totally disconnected graph. Hence $\chi_{\mathrm{ssd}}\left(\mathrm{C}_{\mathrm{n}}\right)=2$ in this case.

From the above cases, we conclude that

$$
\chi_{\text {ssd }}\left(C_{n}\right)=\left\{\begin{array}{ccc}
1 & \text { when } & \mathrm{n}=4,6,8,10 \\
2 & \text { when } & \mathrm{n}=3,5,7 \\
2 & \text { when } & \mathrm{n}=3 \mathrm{k} \text { or } 3 \mathrm{k}+2, \mathrm{k} \geq 3 \\
3 & \text { when } & \mathrm{n}=3 \mathrm{k}+1, \mathrm{k} \geq 4
\end{array}\right.
$$

Theorem 2.4 For path graphs $P_{n}$ of order $n \geq 2, \chi_{s s d}\left(P_{n}\right)=1$.

Proof: Let $P_{n}$ be a path of order $n \geq 2$ with vertex labels $v_{1}, v_{2}, \ldots, v_{n}$. A dominator coloring of $\mathrm{P}_{\mathrm{n}}$ is obtained by coloring the odd subscripted vertices $\mathrm{V}_{1}, \mathrm{~V}_{3}, \mathrm{~V}_{5}, \ldots$ by color 1 and even subscripted vertices $\mathrm{v}_{2}, \mathrm{v}_{4}, \mathrm{v}_{6}, \ldots$, respectively by colors $2,3,4, \ldots$ Removal of color class 1 results in a totally disconnected graph. Hence $\chi_{\text {ssd }}\left(P_{n}\right)$ $=1$. 
Theorem 2.5 For wheel graphs $W_{n}$ of order $n \geq 4$,

$$
\chi_{\text {ssd }}\left(W_{n}\right)=\left\{\begin{array}{lll}
3 & \text { when } n \text { is even } \\
2 & \text { when } n \text { is odd }
\end{array}\right.
$$

Proof: Let $W_{n}$ be a wheel graph of order $n \geq 4$. Let the vertices of the wheel graph $W_{n}, n \geq 4$ be labeled as follows. The vertex at the centre is labeled by $\mathrm{v}_{1}$ and the vertices on the rim be labeled consecutively by $\mathrm{v}_{2}, \mathrm{v}_{3}, \ldots, \mathrm{v}_{\mathrm{n}}$.

A dominator coloring of $W_{n}$ is by coloring $v_{1}$ by color 1 and coloring the vertices in the rim alternatively by colors 2 and 3 from vertex $\mathrm{v}_{2}$.

When $\mathrm{n}$ is odd, the vertices $\mathrm{v}_{\mathrm{n}-1}$ and $\mathrm{v}_{\mathrm{n}}$ are colored by 2 and 3 and when $\mathrm{n}$ is even, the vertices $\mathrm{v}_{\mathrm{n}-2}, \mathrm{v}_{\mathrm{n}-1}$ and $\mathrm{v}_{\mathrm{n}}$ are colored by colors 2, 3 and 4 . Now it is easy to see that the removal of color classes 1 and 2 in the case of $n$ is odd and the color classes 1, 2 and 4 in the case of $n$ is even results in a totally disconnected graph.

From the above two cases, we conclude that

$$
\chi_{\text {ssd }}\left(W_{n}\right)=\left\{\begin{array}{lll}
3 & \text { when } n & \text { is even } \\
2 & \text { when } n & \text { is odd }
\end{array}\right.
$$

Theorem 2.6 For star graphs $S_{n}$ of order $n \geq 3, \chi_{\text {ssd }}\left(S_{n}\right)=1$.

Proof: Let $S_{n}$ be a star graph of order $n \geq 3$. A dominator coloring of $S_{n}$ is by coloring the centre vertex by color 1 and the remaining vertices by color 2 . Removal of color class 1 results in a totally disconnected graph. Hence $\chi_{\text {ssd }}\left(S_{n}\right)=1$.

Theorem 2.7 For complete graphs $K_{n}$ of order $n \geq 2, \chi_{\text {ssd }}\left(K_{n}\right)=n-1$.

Proof: A dominator coloring of $K_{n}$ is by coloring its vertices by colors $1,2,3, \ldots, n$ respectively. Therefore $\chi_{\text {ssd }}\left(K_{n}\right)=n-1$.

Theorem 2.8 For complete bipartite graphs $K_{m, n}, \chi_{s s d}\left(K_{m, n}\right)=1, \forall m$, $\mathrm{n} \geq 2$.

Proof: For $\mathrm{K}_{\mathrm{m}, \mathrm{n}}$, color the vertices in one of the partition by color 1 and the vertices in the other partition by color 2 . This gives a dominator coloring of $\mathrm{K}_{\mathrm{m}, \mathrm{n}}$. Removal of color class either 1 or 2 results in a totally disconnected graph. Hence $\chi_{\mathrm{ssd}}\left(\mathrm{K}_{\mathrm{m}, \mathrm{n}}\right)=1$. 


\section{Strong non-split dominator chromatic number}

In this section, the concept of strong non-split domination [4], is extended to strong non-split dominator chromatic number $\chi_{\text {snd }}(G)$ and find $\chi_{\text {snd }}(G)$ for various classes of graphs.

Definition 3.1 Let $G$ be a graph. Consider its dominator coloring with $\chi_{\mathrm{d}}(\mathrm{G})$ colors. The strong non-split dominator chromatic number of $\mathrm{G}$ is the minimum number of color classes whose removal from $\mathrm{G}$ leaves the remaining graph complete and is denoted by $\chi_{\text {snd }}(G)$.

Example 3.2 Let $G$ be the graph with $V=\left\{v_{1}, v_{2}, v_{3}, v_{4}, v_{5}, v_{6}\right\}$ in Fig. 2. The color classes of $G$ are: $V_{1}=\left\{v_{1}, v_{4}, v_{6}\right\}, V_{2}=\left\{v_{2}\right\}, V_{3}=\left\{v_{3}\right\}$ and $\mathrm{V}_{4}=\left\{\mathrm{v}_{5}\right\}$ Here, $\mathrm{V}-\left\{\mathrm{V}_{1}\right\} \quad$ is complete. Hence $\chi_{\text {snd }}(G)=1$.

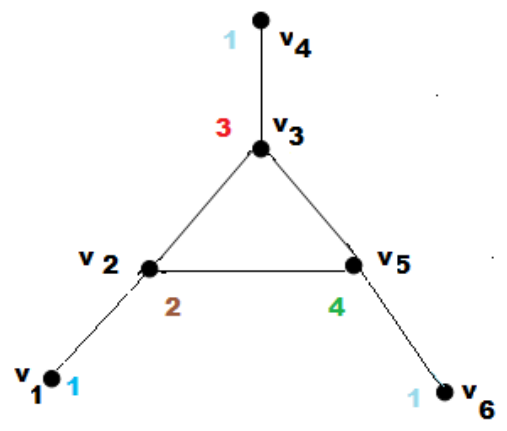

Figure 2

Theorem 3.3 For cycle graphs $C_{n}$,

$$
\chi_{\text {snd }}\left(C_{n}\right)=\left\{\begin{array}{cc}
\lceil n / 3\rceil & \text { when } \mathrm{n}=5,6 \text { or } 3 \mathrm{k}+1, \mathrm{k} \geq 2 \\
\lceil n / 3\rceil+1 & \text { when } \mathrm{n}=3 \mathrm{k}-1 \text { or } 3 \mathrm{k}, \mathrm{k} \geq 3
\end{array}\right.
$$


Proof: Consider the dominator coloring of $C_{n}$ as in Theorem 2.3. The removal of the required number of color classes reduces the graph to either $\mathrm{K}_{1}$ or $\mathrm{K}_{2}$, proving the claim in the theorem.

Note 3.1 For the cycle graph $C_{3}, \chi_{\text {snd }}\left(C_{3}\right)=0$.

Theorem 3.4 For path graphs $P_{n}$ of order $n \geq 3$,

$$
\chi_{\text {snd }}\left(\mathrm{P}_{\mathrm{n}}\right)=\left\{\begin{array}{cc}
\lceil n / 3\rceil & \text { when } n=3,4,5,7 \\
\lceil n / 3\rceil+1 & \text { otherwise }
\end{array}\right.
$$

Proof: Consider the dominator coloring of $\mathrm{P}_{\mathrm{n}}$ as in Theorem 2.4. The result can be seen easily.

Note 3.2 For the path of order 1 and $2, \chi_{\text {snd }}\left(P_{1}\right)=\chi_{\text {snd }}\left(P_{2}\right)=0$.

Theorem 3.5 For wheel graphs $W_{n}$ of order $n \geq 5, \chi_{\text {snd }}\left(W_{n}\right)=2$.

Proof: Consider the dominator coloring of $\mathrm{W}_{\mathrm{n}}$ as in Theorem 2.5. The removal of color classes 2 and 3, for any $n$, results in a complete graph. Hence, $\chi_{\text {snd }}\left(W_{n}\right)=2$.

Note 3.3 For the wheel graph $\mathrm{W}_{4}, \chi_{\text {snd }}\left(\mathrm{W}_{4}\right)=0$.

Theorem 3.6 For the star graph $S_{n}$ of order $n \geq 3, \chi_{\text {snd }}\left(S_{n}\right)=1$.

Proof: Consider the dominator coloring of $S_{n}$ as in Theorem 2.6. Removal of color class 2 results in a complete graph. Hence $\chi_{\text {snd }}\left(S_{n}\right)=1$.

Note 3.4 For complete graphs $K_{n}$ of order $n \geq 1, \chi_{\text {snd }}\left(K_{n}\right)=0$.

\section{Block dominator chromatic number}

In this section, block non-split domination [5] is extended to block dominator chromatic number $\chi_{b d}(G)$ and $\chi_{b d}(G)$ is obtained for various classes of graphs. 
Definition 4.1 Consider a graph $\mathrm{G}$ and its dominator coloring with $\chi_{\mathrm{d}}(\mathrm{G})$ colors. The block dominator chromatic number of $\mathrm{G}$ is the minimum number of color classes to be removed so that the remaining graph is a union of blocks in $G$ and is denoted by $\chi_{\text {bd }}(G)$. We note that for a graph $G$, which is a block, $\chi_{\text {bd }}(G)=0$.

Example 4.2 For the graph in Fig. 2, V- $\left\{\mathrm{V}_{1}\right\}$ is a block. Hence $\chi_{\mathrm{bd}}(\mathrm{G})$ $=1$.

Note 4.1 We observe the following facts:

- For the cycle $C_{n}$ of order $n \geq 3, \chi_{b d}\left(C_{n}\right)=0$.

- For the wheel graph $W_{n}$ of order $n \geq 4, \chi_{b d}\left(W_{n}\right)=0$.

- For the complete graph $K_{n}$ of order $n \geq 2, \chi_{b d}\left(K_{n}\right)=0$.

- For the bipartite graph $\mathrm{K}_{\mathrm{m}}$, $\mathrm{n}$ of order $\mathrm{m}, \mathrm{n} \geq 2$, we have $\chi_{b d}\left(K_{m, n}\right)=0$.

\section{Cycle non-split dominator chromatic number}

In this section, the notion of cycle non-split domination is extended to cycle non-split dominator chromatic number $\chi_{\mathrm{cnd}}(\mathrm{G})$ and $\chi_{\mathrm{cnd}}(\mathrm{G})$ is obtained for $\mathrm{W}_{\mathrm{n}}$.

Definition 5.1 Consider a graph $\mathrm{G}$ and its dominator coloring with $\chi_{\mathrm{d}}(\mathrm{G})$ colors. The cycle non-split dominator chromatic number of $\mathrm{G}$ is the minimum number of color classes that should be removed so that the remaining graph is a cycle and is denoted by $\chi_{\mathrm{cnd}}(\mathrm{G})$.

Example 5.2 Consider the graph in Fig. 2, and its corresponding color classes. We observe that $V-\left\{V_{1}\right\}$ is a cycle. Hence $\chi_{\text {cnd }}(G)=1$.

Theorem 5.3 For the wheel graph $W_{n}$ of order $n \geq 4, \chi_{\mathrm{cnd}}\left(W_{n}\right)=1$.

Proof: Consider the dominator coloring of $W_{n}$ as in Theorem 2.5. It can be seen that the removal of color class 1 for any $n$ results in a cycle graph. Hence $\chi_{\mathrm{cnd}}\left(\mathrm{C}_{\mathrm{n}}\right)=1$. 
Theorem 5.4 For the wheel graph $W_{n}$ of order $n \geq 4$, we have $\chi_{\text {cnd }}\left(\mathrm{W}_{\mathrm{n}}\right)+\Delta\left(\mathrm{W}_{\mathrm{n}}\right)=\mathrm{n}$.

Observation 5.1 For the complete graph $C_{n}, \chi_{\text {cnd }}\left(K_{n}\right)=n-3$.

Observation 5.2 For the cycle graph $C_{n}, \chi_{c n d}\left(C_{n}\right)=0$.

Observation 5.3 For the complete bipartite graph $K_{m, n}, \chi_{c n d}\left(K_{m}, n\right)=$ 0 , when $\mathrm{m}=\mathrm{n}=2$.

\section{Path non-split dominator chromatic number}

In this section, we extend the notion of path non-split domination, to path non-split dominator chromatic number $\chi_{\text {pnd }}(G)$ and obtain $\chi_{\text {pnd }}(G)$ for various classes of graphs.

Definition 6.1 Consider a graph $\mathrm{G}$ and its dominator coloring with $\chi_{\mathrm{d}}(\mathrm{G})$ colors. The Path non-split dominator chromatic number of $\mathrm{G}$ is the minimum number of color classes that should be removed so that the remaining graph is a path and is denoted by $\chi_{\text {pnd }}(G)$.

Example 6.2 Let $G$ be the graph with $V=\left\{v_{1}, v_{2}, v_{3}, v_{4}, v_{5}, v_{6}, v_{7}, v_{8}\right.$, $\left.v_{9}\right\}$ in Figure 4.

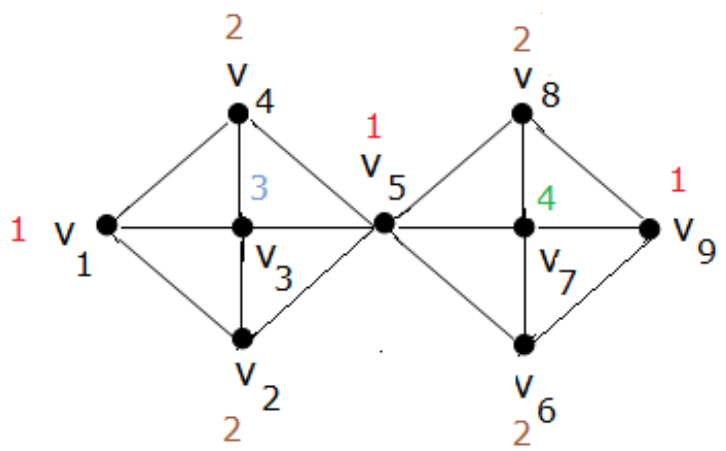

Figure 4

The color classes of $\mathrm{G}$ are, $\mathrm{V}_{1}=\left\{\mathrm{v}_{1}, \mathrm{v}_{5}, \mathrm{v}_{9}\right\}, \mathrm{V}_{2}=\left\{\mathrm{v}_{2}, \mathrm{v}_{4}, \mathrm{v}_{6}, \mathrm{v}_{8}\right\}, \mathrm{V}_{3}=$ $\left\{\mathrm{v}_{3}\right\} \& \mathrm{~V}_{4}=\left\{\mathrm{v}_{7}\right\}$.

Here, $V-\left\{V_{2}\right\}$ is a path. Hence $\chi_{\text {pnd }}(G)=1$. 
Theorem 6.3 For the cycle graph $C_{n}$ of order, $\chi_{\text {pnd }}\left(C_{n}\right)=1$, when $n=$ $3,5,6,7, \ldots$

Proof: Consider the dominator coloring of $C_{n}$ as in Theorem 2.3. The removal of the last color class reduces the graph to a path of order $n-1$. Hence $\chi_{\text {pnd }}\left(C_{n}\right)=1$.

Theorem 6.4 For the wheel graph $W_{n}$ of order $n \geq 4$,

$$
\chi_{\text {pnd }}\left(W_{n}\right)=\left\{\begin{array}{lc}
2 & \text { when } \mathrm{n}=4,6,7,8,9, \ldots \\
1 & \text { when } \mathrm{n}=5
\end{array}\right.
$$

Proof: Consider the dominator coloring of $\mathrm{W}_{\mathrm{n}}$ as in Theorem 2.5. It can be seen that the removal of color class 2 and 3 when $n=4,6,7$, ... results in a path. When $n=5$, removal of either the color class 2 or 3 results in a path.

Therefore, we have

$$
\chi_{\text {pnd }}\left(W_{n}\right)=\left\{\begin{array}{lc}
2 & \text { when } n=4,6,7,8,9, \ldots \\
1 & \text { when } n=5
\end{array}\right.
$$

Theorem 6.5 For the complete graph $K_{n}$ of order $n \geq 2, \chi_{\text {pnd }}\left(K_{n}\right)=$ $\mathrm{n}-2$.

Proof: A dominator coloring of $K_{n}$, is by coloring its vertices by colors $1,2,3, \ldots, \mathrm{n}$ respectively. Removal of $\mathrm{n}-2$ color classes results in a path of order 2 . Therefore $\chi_{\text {pnd }}\left(K_{n}\right)=n-2$. 


\section{References}

[1] S. Arumugam, On dominator coloring in graphs, IWGC, BDU, 2010.

[2] V.R. Kulli, Theory of Domination in Graphs, Vishwa International Publication, Gulbarga, India, 2010.

[3] V.R. Kulli and B. Janakiram, The strong split domination number of a graph, Acta Ciencia Indica, 32M (2006) 715-720.

[4] V.R. Kulli and B. Janakiram, The strong non-split domination number of a graph, Internat. J. Management Systems, 19 (2003) 145156.

[5] V.R. Kulli and B. Janakiram, The block non-split domination number of a graph, Internat.J. Management Systems, 20 (2004) 219228. 